\title{
The shorter multidrug-resistant tuberculosis treatment regimen in Singapore: are patients from South-East Asia eligible?
}

To the Editor:

Multidrug-resistant tuberculosis (MDR-TB) is a major public health challenge, with an estimated 480000 new cases emerging globally each year [1]. Treatment success rates using the 20-month conventional World Health Organization (WHO) regimen have been low, being about 52\% in 2013 [1]. Observational studies in Bangladesh and several African countries have shown success rates of $84 \%$ using a shorter regimen [2-4]. Based on these studies, in May 2016 the WHO conditionally recommended a standardised, shorter 9-12-month regimen for MDR pulmonary tuberculosis (PTB), comprising a 4-6-month intensive phase with a combination of kanamycin, moxifloxacin, prothionamide, clofazimine, pyrazinamide, high-dose isoniazid and ethambutol, followed by a 5-month continuation phase containing moxifloxacin, clofazimine, ethambutol and pyrazinamide [5]. Confirmed resistance (except to isoniazid) or suspected ineffectiveness to a drug in this regimen is one of the exclusion criteria [5].

Several researchers have highlighted the ambiguity of the eligibility criteria and expressed doubts as to the widespread applicability of this regimen [6]. Publications from Europe have reported eligibility rates ranging from $6.2 \%$ to $14 \%$ [7-10]. Data from Pakistan and Brazil showed more optimistic eligibility rates of $50 \%$ or higher $[11,12]$. A multicountry population-based survey showed that, although pyrazinamide resistance was significantly associated with rifampicin resistance, this drug might still be effective in 19-63\% of patients with rifampicin-resistant TB, and that the worrisome high level of ofloxacin resistance in Pakistan (probably reflecting extensive, unregulated use of fluoroquinolones in some parts of Asia) was tempered by the negligible levels of resistance to fourth-generation fluoroquinolones documented in all survey sites [13]. To our knowledge, there are to date no publications on the eligibility of this regimen among patients with MDR-PTB from South-east Asia, India and China.

Singapore, a country with an intermediate TB incidence in South-east Asia, receives migrant workers and medical tourists from countries with high TB incidence in the region. Since 2008, foreign-born TB cases have accounted for slightly above $40 \%$ of the country's TB burden. MDR-TB has been reported in $3 \%$ of foreign-born cases of PTB and $0.3 \%$ of PTB cases born in Singapore or Malaysia [14].

The diagnosis of MDR/rifampicin-resistant PTB in developing countries is often based solely on the Xpert MTB/Rif assay (Cepheid, Sunnyvale, USA), and many of these countries lack quality-assured laboratory capacity to perform full phenotypic drug susceptibility testing (DST). Information collected by the WHO on DST reporting for fluoroquinolones and second-line injectables indicate that, among MDR/ rifampicin-resistant patients with TB notified to the authorities in 2015, only $36 \%$ were reported to have DST to these drug classes, with the lowest coverage being in the WHO Western Pacific and South-east Asia regions [1]. In developing countries where the highest burden of MDR-TB exists, the availability of DST for the other drugs in the WHO shorter regimen (i.e., pyrazinamide, ethionamide/prothionamide and clofazimine) is not known. Although the WHO-endorsed Genotype MTBDRsl versions 1 and 2 (Hain LifeScience, Nehren, Germany) detect genotypic resistance to the fluoroquinolones and second-line injectables, there are currently no commercial assays to detect genotypic resistance to pyrazinamide, ethionamide or clofazimine.

@ERSpublications

About $30 \%$ of MDR pulmonary-TB patients in a SE Asian (Singaporean) sample are eligible for the WHO shorter regimen http://ow.ly/KF3330dMSVs

Cite this article as: Chee CBE, KhinMar K-W, Sng L-H, et al. The shorter multidrug-resistant tuberculosis treatment regimen in Singapore: are patients from South-East Asia eligible? Eur Respir J 2017; 50: 1700753 [https://doi.org/10.1183/13993003.00753-2017]. 
Singapore is served by two mycobacterial culture laboratories, the Central TB Laboratory at the Singapore General Hospital and the Microbiology Laboratory of the National University Hospital. Both laboratories routinely perform first-line DST to isoniazid, rifampicin, ethambutol and streptomycin for all patients with positive Mycobacterium tuberculosis complex (MTC) isolates. DST to kanamycin, ofloxacin and ethionamide is routinely performed on isolates resistant to rifampicin and/or isoniazid. DST to pyrazinamide, para-amino salicylic (PAS), clofazimine and capreomycin are performed on request. The critical concentrations used for each of the drugs are as follows: rifampicin $1.0 \mu \mathrm{g} \cdot \mathrm{mL}^{-1}$, isoniazid $0.1 \mu \mathrm{g} \cdot \mathrm{mL}^{-1}$, ethambutol $5.0 \mu \mathrm{g} \cdot \mathrm{mL}^{-1}$, kanamycin $2.5 \mu \mathrm{g} \cdot \mathrm{mL}^{-1}$, ofloxacin $2.0 \mu \mathrm{g} \cdot \mathrm{mL}^{-1}$, ethionamide $5.0 \mu \mathrm{g} \cdot \mathrm{mL}^{-1}$ and clofazimine $0.5 \mu \mathrm{g} \cdot \mathrm{mL}^{-1}$. All positive MTC culture and DST results are captured in the National TB Registry via electronic linkage to both mycobacterial laboratories because TB is a notifiable disease in Singapore. Patient demographic data, country of origin and type of case (new/previously treated) are captured in the National TB Registry.

Using data from the registry, we analysed the phenotypic DST results to the constituent drugs (except isoniazid) of the shorter WHO regimen of all MDR-PTB cases diagnosed in Singapore from 2002 to 2016 to determine their eligibility for this regimen. Ethics approval was not required, as this was registry data analysed in aggregate form.

During the study period, there were 270 patients with MDR-PTB (202 new and 68 previously treated cases), of whom 49 were born in Singapore, five were born in Malaysia and 216 (80\%) were born outside of Singapore/Malaysia. The latter group comprised patients from Indonesia $(n=76)$, Myanmar $(n=56)$, China ( $n=37)$, Philippines $(n=18)$, Vietnam $(n=15)$, India $(n=7)$ and Bangladesh $(n=4)$, with one patient each from Saudi Arabia, Tonga and Russia. Nine patients (3.3\%) were extensively drug-resistant (XDR) and $29(10.7 \%)$ were pre-XDR cases.

The patients from Saudi Arabia, Tonga and Russia (none of whom had XDR) were excluded from our analysis. For the remaining 267 patients, phenotypic DST results to ethambutol were available for all; to kanamycin and ethionamide in 266 (99.6\%), to ofloxacin in 261 (97.8\%), to pyrazinamide in $165(61.8 \%)$ and to clofazimine in $105(39.3 \%)$ (table 1$)$. Of those tested, $4.1 \%(11 / 266)$ were resistant to kanamycin,

TABLE 1 Number and percentage of cases resistant to each of the six individual drugs in the shorter World Health Organization regimen, resistant to at least one of the six drugs and resistant to at least one of the five drugs excluding ethambutol, classified according to country of origin

\begin{tabular}{|c|c|c|c|c|c|c|c|c|c|}
\hline Drug & $\begin{array}{l}\text { Bangladesh } \\
\qquad(n=4)\end{array}$ & $\begin{array}{l}\text { China } \\
\text { (n=37) }\end{array}$ & $\begin{array}{l}\text { India } \\
(n=7)\end{array}$ & $\begin{array}{c}\text { Indonesia } \\
(n=76)\end{array}$ & $\begin{array}{c}\text { Myanmar } \\
(n=56)\end{array}$ & $\begin{array}{l}\text { Philippines } \\
\text { (n=18) }\end{array}$ & $\begin{array}{c}\text { Vietnam } \\
(n=15)\end{array}$ & $\begin{array}{l}\text { Singapore/ } \\
\text { Malaysia } \\
(n=54)\end{array}$ & $\begin{array}{c}\text { Total } \\
(n=267)\end{array}$ \\
\hline Tested & $4(100)$ & 37 (100) & 7 (100) & $76(100)$ & $56(100)$ & $18(100)$ & 15 (100) & 54 (100) & 267 (100) \\
\hline Resistant & 3 (75.0) & $13(35.1)$ & $5(71.4)$ & 38 (50.0) & $34(60.7)$ & $2(11.1)$ & $6(40)$ & $15(27.8)$ & 116 (43.4) \\
\hline \multicolumn{10}{|l|}{ Pyrazinamide } \\
\hline \multicolumn{10}{|l|}{ Ethionamide } \\
\hline Tested & $4(100)$ & 37 (100) & 7 (100) & 75 (98.7) & $56(100)$ & 18 (100) & 15 (100) & 54 (100) & 266 (99.6) \\
\hline Resistant & $0(0.0)$ & 14 (37.8) & $0(0.0)$ & $17(22.7)$ & 8 (14.3) & $8(44.4)$ & 2 (13.3) & $26(48.1)$ & 75 (28.2) \\
\hline \multicolumn{10}{|l|}{ Kanamycin } \\
\hline Tested & $4(100)$ & 37 (100) & $7(100)$ & 75 (98.7) & $56(100)$ & $18(100)$ & $15(100)$ & $54(100)$ & $266(99.6)$ \\
\hline Resistant & $1(25.0)$ & $1(2.7)$ & $0(0.0)$ & $7(9.3)$ & $0(0.0)$ & $0(0.0)$ & 2 (13.3) & $0(0.0)$ & $11(4.1)$ \\
\hline Resistant & $0(0.0)$ & $0(0.0)$ & $0(0.0)$ & $3(16.7)$ & $1(4.3)$ & $0(0.0)$ & $0(0.0)$ & $0(0.0)$ & 4 (3.8) \\
\hline $\begin{array}{l}\text { Resistant to at least } \\
\text { one of the above } \\
\text { six drugs }\end{array}$ & $3(75.0)$ & 23 (62.2) & $6(85.7)$ & 57 (75.0) & 40 (71.4) & $10(55.6)$ & 12 (80.0) & 39 (72.2) & 190 (71.2) \\
\hline $\begin{array}{l}\text { Resistant to at least } \\
\text { one of the above five } \\
\text { drugs excluding } \\
\text { ethambutol }\end{array}$ & $1(25.0)$ & $20(54.1)$ & $2(28.6)$ & 44 (57.9) & 30 (53.6) & $9(50.0)$ & 8 (53.3) & 28 (51.9) & 142 (53.2) \\
\hline
\end{tabular}

Data are presented as $\mathrm{n}(\%)$ unless otherwise noted. 
$28.2 \%(75 / 266)$ to ethionamide, $13.4 \%(35 / 261)$ to ofloxacin, $43.4 \%(116 / 267)$ to ethambutol, $43.6 \%(72 /$ $165)$ to pyrazinamide and $3.8 \%(4 / 105)$ to clofazimine. Of the 267 cases, $190(71.2 \%)(137 / 200(68.5 \%)$ of the new cases and $53 / 67(79.1 \%)$ of the previously treated cases) were resistant to at least one of the six constituent drugs of the WHO shorter regimen. Excluding ethambutol (in view of the lower reliability of DST for this drug [15]), 142 (53.2\%) were resistant to at least one of the other five drugs. Only $22(8.2 \%)$ showed confirmed susceptibility to all six drugs.

Among MDR-PTB patients from Indonesia and Myanmar, who together accounted for $60 \%$ of Singapore's foreign-born cases of MDR-TB, 75\% and 71.4\%, respectively, were resistant to at least one of the six drugs in the WHO shorter regimen. Patients with MDR-PTB from the Philippines and Singapore/Malaysia had considerably lower rates of resistance to ethambutol (11.1\% and $27.8 \%$ respectively) and pyrazinamide (30\% and $6.8 \%$ respectively), but higher rates of ethionamide resistance ( $44.4 \%$ and $48.1 \%$ respectively) compared with the patients from the other countries.

Ofloxacin resistance was significantly more likely among those previously treated $(11.2 \%$ versus $25 \%$, $\mathrm{p}=0.007)$. There was no significant difference in the rates of resistance to kanamycin $(3.0 \%$ versus $7.6 \%$, $\mathrm{p}=0.10)$, ethionamide $(28.5 \%$ versus $27.3 \%, \mathrm{p}=0.85)$, ethambutol $(42.0 \%$ versus $47.8 \%, \mathrm{p}=0.41)$, pyrazinamide $(41.6 \%$ versus $50.0 \%, \mathrm{p}=0.35)$, and clofazimine $(2.4 \%$ versus $10.0 \%, \mathrm{p}=0.11)$ between new and previously treated cases.

Our study has several limitations. Only 62\% and 39\% of cases had DST performed to pyrazinamide and clofazimine, respectively. The small number of cases in our cohort may not be representative of patients with MDR-TB in the respective countries of origin. Information pertaining to new or previously treated TB and country of origin was subject to the accuracy of the notifying physician's submission to the registry.

Our study shows that only about 30\% of patients with MDR-PTB diagnosed in this sample from South-east Asia would be eligible for the WHO shorter MDR-TB treatment regimen. Ignoring ethambutol resistance, approximately $50 \%$ of these cases would be eligible. However, in the absence of individual full DST results, we advise caution in the use of this regimen in patients from the South-east Asian countries represented in our study.

Cynthia B.E. Chee ${ }^{1,2}$, Kyi-Win KhinMar ${ }^{2}$, Li-H. Sng ${ }^{3}$, Roland Jureen ${ }^{4}$, Jeffery Cutter ${ }^{5}$, Vernon J.M. Lee ${ }^{5}$ and Yee-Tang Wang ${ }^{1,2}$

${ }^{1}$ Respiratory Medicine, Tan Tock Seng Hospital, Singapore, Singapore. ${ }^{2}$ Tuberculosis Control Unit, Tan Tock Seng Hospital, Singapore, Singapore. ${ }^{3}$ Dept of Pathology, Singapore General Hospital, Singapore, Singapore. ${ }^{4}$ National University Health System (NUHS), Singapore, Singapore. ${ }^{5}$ Communicable Diseases Division, Ministry of Health, Singapore, Singapore.

Correspondence: C.B.E. Chee, Respiratory Medicine, Tan Tock Seng Hospital, 11 Jalan Tan Tock Seng, Singapore 308433, Singapore. E-mail: cynthia_chee@ttsh.com.sg

Received: April 112017 | Accepted after revision: May 062017

Conflict of interest: None declared.

\section{References}

1 Global tuberculosis report 2016. Geneva, World Health Organization, 2016. http://apps.who.int/iris/bitstream/ 10665/250441/1/9789241565394-eng.pdf?ua=1

2 Van Deun A, Maug AKJ, Salim MAH, et al. Short, highly effective, and inexpensive standardized treatment of multidrug-resistant tuberculosis. Am J Respir Crit Care Med 2010; 182: 684-692.

3 Kuaban C, Noeske J, Rieder HL, et al. High effectiveness of a 12-month regimen for MDR-TB patients in Cameroon. Int J Tuberc Lung Dis 2015; 19: 517-524.

4 Piubello A, Harouna SH, Souleymane MB, et al. High cure rate with standardized short-course multidrug-resistant tuberculosis treatment in Niger: no relapses. Int J Tuberc Lung Dis 2014; 18: 1188-1194.

5 Falzon D, Schunemann H, Harausz E, et al. World Health Organization treatment guidelines for drug-resistant tuberculosis, 2016 update. Eur Respir J 2017; 49: 1602308.

6 Varaine F, Guglielmetti L, Huerga $\mathrm{H}$, et al. Eligibility for the shorter multidrug resistant tuberculosis regimen: ambiguities in the World Health Organization recommendations. Am J Respir Crit Care Med 2016; 194: 1028-1029.

7 Balabanova Y, Fiebig L, Ignatyeva O, et al. Multidrug-resistant TB in Eastern region of the EU: is the shorter regimen an exception or a rule? Thorax 2017; in press [https://doi.org/10.1136/thoraxjnl-2016-209841].

8 Lange C, Duarte R, Frechet-Jachym M, et al. Limited benefit of the new shorter multidrug-resistant tuberculosis regimen in Europe. Am J Respir Crit Care Med 2016; 194: 1029-1031.

9 van der Werf MJ, Hollo V, Kodmon C, et al. Eligibility for shorter treatment of multidrug-resistant tuberculosis in the European Union. Eur Respir J 2017; 49: 1601992.

10 Sotgiu G, Tiberi S, D'Ambrosio L, et al. Faster for less: the new shorter regimen for multidrug-resistant tuberculosis. Eur Respir J 2016; 48: 1503-1507.

11 Jarvaid A, Ahmad N, Khan AH, et al. Applicability of the World Health Organization recommended new shorter regimen in a multidrug-resistant tuberculosis high burden country. Eur Respir J 2017; 49: 1601967.

12 Dalcolmo M, Gayoso R, Sotgiu G, et al. Resistance profile of drugs composing the "shorter" regimen for multidrug-resistant tuberculosis in Brazil, 2000-2015. Eur Respir J 2017; 49: 1602309. 
13 Zignol M, Dean AS, Alikhanova N, et al. Population-based resistance of Mycobacterium tuberculosis isolates to pyrazinamide and fluoroquinolones: results from a multicountry surveillance project. Lancet Infect Dis 2016; 16: $1185-1192$.

14 Communicable diseases surveillance in Singapore 2015. Singapore, Singapore Ministry of Health, 2016. www.moh. gov.sg/content/dam/moh_web/Publications/Reports/2016/Full\%20Version.pdf

15 Yakrus MA, Driscol J, McAlister A, et al. Molecular and growth-based drug susceptibility testing of Mycobacterium tuberculosis complex for ethambutol resistance in the United States. Tuberc Res Treat 2016; 2016: 3404860. 